\title{
XXI.
}

\section{Casuistische Mittheilungen}

aus der

\section{chirurgischen Klinik des Herrn Geheimen Obermedicinalrath Prof. Dr. Bardeleben. \\ Von}

Stabsarzt Dr. Alberti.

\section{Eine Unterbindung der gemeinsamen Schenkelvene am Poupart'schen Band. Heilung.}

E. Harms, Näherin, 20 Jahre alt, wurde am 9. Februar 1883 wegen eines Ulcus am Orificium urethrae in die Charité anfgenommen. Ein sich allmählich entwickelnder, linksseitiger Inguinalbubo abscedirte, und schritt man am 10. März zur Incision und Exstirpation der geschwollenen und zerfallenden Drüsen. Ein schräg von oben und aussen nach unten und innen verlaufender Schnitt legte ein in eiteriger Einschmelzung vorgeschrittenes Drüsenpacket frei, dessen einzelne Drüsen sich leicht mit den Fingern ausschälen liessen. Dies Herauspräpariren war von dem ordinirenden Arat der betreffenden Abtheilung einem Unterarzte zu seiner Uebung überlassen worden; Ersterer controlirte gerade die Narkose, als der junge Operateur ein in der Tiefe noch festsitzendes Drüsenpacket mittelst einer Cooperschen Scheere ausschnitt. Sogleich sprudelte ein mächtiger Blutstrom hervor, der bei jedem Versuch, die sofort unternommene Digitalcompression nachzulassen, um die verletzte Stelle zu Gesicht zu bekommen, herausquoll. Als ich die Kranke etwa 12 Minuten nach der Verletzung sah, war diese recht anämisch. Es wurde nun ober- und unterhalb der verletzten Stelle, zur genaueren Recognoscirung derselben, comprimirt; doch sickerte noch etwas Blut aus, und zwar - wie deutlich zu sehen war - aus der verletzten Vena femoralis. In dem zuletzt ausgeschnittenen Drüsenpacketchen war ein breites, schräg durehschnittenes Venenlumen sicbtbar - wohl das der durchschnittenen Vena saphena. Ein Gefässstumpf vom centralen Ende der letzteren, an der verletzten Stelle der Vena femoralis, war nicht zu entdecken; deshalb wurde - namentlich in Rücksicht auf die schon erhebliche Anämie - von jedem Versuch einer seitlichen Ligatur Abstand genommen und sogleich zur doppelten Ligatur der Vena femoralis geschritter.

Unter dauernder Compression der Venenwunde erweiterte ich den Hautschnitt, entfernte von der Gefässscheide die noch zurückgebliebenen Drüsen- 
reste, eröffnete die Gefässscheide und legte erst peripherisch von der Venenwunde eine starke Catgutligatur um die Vene. Diese hatte - was vorauszusehen war - keinen sichtbaren Einfluss auf die Stärke der Blutung, wie die momentan nachgelassene Compression ergab. Die Blutung stand erst, als die centrale Ligatur möglichst hoch oben, direct im Schenkelring, angelegt wurde. Es zeigte sich nun ein fast $1 \mathrm{Cm}$. langer Schnitt, oder vielmehr ein schmaler, längsovaler Defect in der Wand der Ven. femoralis, zugleich an dessen unterem Ende ein kleiner spitzer Rest eines Seitenastes der Wand der durchschnittenen V. saphena.

Während der Operation wiederholte Berieselung der Wunde mit Sublimatlösung $(0,1: 100,0)$; darnach Naht der Hautwunde bis auf die Drainöffnung, kurzer Drain, antiseptischer Verband mit in gleichstarker Sublimatlösung angefeuchteten Compressen, Einwickelnng des ganzen Beins bis zur Wunde hin mit einer Flanellbinde und verticale Suspension des Beins nach der sogleich erfolgten Verlegung der Patientin zur chirurgischen Abtheilung. Bald nach angelegtem Verband leichte Cyanose des Fusses, die aber nicht länger als $1 \frac{1}{2}$ Stunden anhält. Nachmittags ist dieselbe geschwunden, kein Oedem des Fusses, Gefühl desselben nicht gestört; grosse Mattigkeit und Unruhe, Temp. 37,0, Puls 110, von niedriger Welle.

11. März. In der Nacht wenig Schlaf; öfters Schmerzen im linken Bein, die sich am Vormittag noch steigern. Temp. 37,5, Puls 120. Nach Entfernung der Flanellbinde zeigt das linke Bein weder Cyanose, noch Oedem; Sensibilität unverändert; auch in Motilität (stärkere Bewegungen werden absichtlich vermieden) anscheinend kein Unterschied gegen das rechte Bein. Verband intact. Nachmittags Schüttelfrost, Temp. 40,1, Puls 128. Kopfschmerz, grosse Mattigkeit; Gefühl von Taubsein in den Zehen, ziehende Schmerzen in der linken Wade. Am Bein selbst nichts Abnormes zu sehen.

12. März. Temp. früh 38,6, Puls 126 ; Abends 38,2, Puls 120. Keine wesentliche Veränderung; subjectiv besseres Befinden.

13. März. Temp. früh 38,6, Puls 100 ; Abends 39,8, Puls 100. Nachts wenig Schlaf. Schmerzen, abwechselnd mit Gefühl von Taubsein, in der linken Wade. Sensibilität des linken Unterschenkels erheblich herabgesetzt: oberfächliche Nadelstiche werden gar nicht, Stiche bis zur Tiefe von gut $1 / 2 \mathrm{Cm}$. nur schwach empfunden, zugleich sehr ungenau localisirt. Am Knie und Oberschenkel normales Gefühl. Kein Unterschied der Motilität, sowie der elektrischen Erregbarkeit der Muskeln gegen das rechte Bein. Nach Abnahme des Verbandes zeigt die zum grossen Theil per primam geheilte Wunde geringe Secretion, doch oberhalb eine, anscheinend erysipelatöse Röthung und Schwellung der Haut, die sich strahlig nach oben und aussen bis zur Höhe der Spina Ilei ant. sup. erstreckt, scharf gegen die normale Haut absetzt und bei Berührung ausserordentlich empfindlich ist. Unterhalb der Wunde ist die Haut normal. - Wiederum feuchter Sublimatverband, welcher auch die gerötheten Hautpartien bedeckt.

14. März. Früh Temp. 38,4, Puls 120; Abends Temp. 39,1, Puls 120. Die Röthung am Abdomen unverändert, nicht weiter vorgeschritten. GefühI von "Taub- und Abgestorbensein " im linken Bein; Sensibilitätsstörung wie gestern.

15. März. Früh Temp. 38,0, Puls 96 ; Abends Temp. 39,2, Puls 90. Röthung und Schwellung, vor Allem die Schmerzhaftigkeit der gerötheten 
Stellen haben abgenommen. Wunde, von gutem Aussehen, secernirt wenig. Meteoristische Auftreibung des Leibes, starke Flatulenz, 1 diunner Stuhl. D i e Sensibilität des linken Unterschenkels ist wieder normal.

In den nächsten Tagen fiel das Fieber, unter morgendlichen Remissionen meist bis 38,0 und darunter; vom 19. März, also vom 9. Krankbeitstage, an war Patientin fieberfrei. Bereits am 18. März war die Röthung am Abdomen fast ganz geschwunden; doch blieben noch drei ganz circumscripte, gut fingerdicke und $4-5 \mathrm{Cm}$. lange, stark geröthete und sehr schmerzhafte Stellen dicht über der Wunde und von dieser aus sich strahlig nach oben erstreckend fünf Tage lang bestehen; Abschuppung der Haut trat nicht ein. Während dieser Tage hatte Patientin fast andauernd mässige Tympanie des Leibes mit täglich mehreren dünnen Stuhlentleerungen. Zugleich stellte sich ein diffuser Bronchialkatarrh mit reichlichem Auswurf, der einige Male blutig gefärbt war, ein, schwand aber gleichzeitig mit den Darmerscheinungen. Die Tympanie des Leibes trat in den folgenden Wochen noch wiederholt - und zwar obne irgend welche nachweisbare Diätfehler jedesmal mit grosser Empfindlichkeit der linken Unterbauchgegend auf und schwand meist schon innerhalb weniger Stunden nach 2 oder 3 dünnen, schmerzbaften Entleerungen. Das Allgemeinbefinden blieb dabei gut; doch besserte sich die Anämie nur langsam. Die Wunde heilte ohne weitere Störung. In den ersten Wochen klagte Patientin noch öfters über Schmerzen und "Ziehen " in der linken Wade; Störungen der Sensibilität stellten sich nicht mehr ein. Am 30. Krankheitstage verliess Patientin zum ersten Mal das Bett und am 22. April - das war am 43. Krankheitstage - geheilt die Anstalt.

Sie siedelte daranf nach Breslan über und berichtete von dort Ende Juli 1883, dass sie nach Anstrengangen grosse Ermuidung des linken Beins bekäme; auch stellten sich noch öfters Schmerzen in der linken Bauchseite ein, „so wie diese in der Charité aufgetreten". Doch schrieb sie Ende December 1883: "Ich bin jetzt schon seit Monaten so wohl, als wenn ich keine unterbundene Vene hätte; von Leibschmerzen spüre ich nichts mehr und merke ich beim Gehen nicht das Mindeste; nur fühle ich bei längerem Stehen Schwäche im linken Bein, doch keine Schmerzen, noch Beschwerden."

Die grosse Mehrzahl der deutschen Chirurgen neigte sich bis in die neueste Zeit der Meinung hin, dass bei Verletzung der V. femoralis unter dem Poupart'schen Bande nicht die Vene allein, sondern die Arterie oder Arterie und Vene zu unterbinden sei, da der isolirten Unterbindung der Vene am leichtesten Gangrän des Beines folgen wïrde. Die anatomischen Untersuchungen W. Braune's ${ }^{1}$, welcher nachwies, dass die Schenkelvene zwar Anastomosen mit den Beckenvenen hat, in diesen aber die Klappen in der der Schenkelvene zugewandten Hälfte der Anastomosen so gestellt sind, dass in ihnen wohl ein Blutzufluss nach der Schenkelvene möglich, umge-

1) Die Oberschenkelvene des Menschen in anatomischer nnd klinischer Beziehung. Leipzig 1571 . 
kehrt aber Rückfluss des Blutes durch diese, wenn nicht ganz unmöglicb, so doch aufs Aeusserste erschwert sei, bestärkten diese Meinung. H. Braun ${ }^{1}$ hat in seiner eingehenden Abhandlung eine Reihe gewichtigster Stimmen angeführt, welche sich gegen die isolirte Unterbindung der Schenkelvene ausgesprochen haben, und bei genauer Sammlung der veröffentlichten Fälle, in denen diese unternommen worden, nur 17 zusammenstellen können. Erst Rose ${ }^{2}$ ) und dann jüngst, fast zu gleicher Zeit mit Braun empfahlen Maas ${ }^{3}$ ) und v. Bergmann ${ }^{4}$ ), theils auf Grund von glïcklich verlaufenen Fällen, theils von Leichenexperimenten, welche, von den physiologischen Circulations- und Druckerscheinungen in den Venen Lebender ausgehend, zu ganz anderen Resultaten führten, als die von Braune auf Grund der von ihm gefundenen anatomischen Thatsachen angestellten Versuche, die isolirte Unterbindung der Schenkelvene. Braune ${ }^{5}$ ) hatte nach Unterbindung der Vena femor. am Poupart'schen Bande Injectionsflissigkeiten, theils von ihren Aesten, theils von der Art. femor. aus in dieselbe einfliessen lassen und gefunden, dass bei schwachem Druck nichts von den Flüssigkeiten in die Beckenvenen eindrang und erst bei stärkerem Druck die Klappen in den Anastomosen überwunden wurden. Nun steigt aber am Lebenden nach Unterbindung der Vene der Druck in derselben ausserordentlich, ja er wird binnen Kurzem dem im zuführenden Arterienrohr - d. i. p. p. einem Druck von 110-120 Mm. Hg. - gleichkommen, einem Druck, welcher in den meisten von $\mathrm{Braun}{ }^{6}$ ) an Leichen angestellten Controlversuchen geniggte, die Injectionsflussigkeit durch die Anastomosen der V. femoralis in die Beckenvenen zu treiben.

Die Resultate, welche Versuche mit Eingiessen von Flüssigkeiten in die Venen von Leichen ergeben, sind gewiss nur mit grosser Reserve auf die Verhältnisse am Lebenden anzuwenden. Es kommen hier alle die Kräfte in Betracht, welche auf die Blutbewegung in den Venen von Einfluss sind, so die Trieb- und Saugkraft des Herzens, die Bewegung der Muskeln, die Aspiration durch den Brust-

1) Die Unterbindung der Schenkelvene am Poupart'schen Band. Archiv für klin. Chirurgie. Bd. XXVIII. S. 610.

2) Ueber Stichwunden der Schenkelgefässe und ihre sicherste Behandlung. Volkmann's ges. klin. Vorträge. 1875. Nr. 92.

3) Die Circulation der unteren Extremität. Diese Zeitschrift. 1852. Bd. XVII. S. 197 .

4) Die isolirte Unterbindung der Vena fem. commun. Festschrift zur Feier des 300 jähr. Bestehens der Julius-Maximilian's Universität zu Würzburg. 1892. S. 215.

$\begin{array}{ll}\text { 5) 1. c. S. } 12-14 . & \text { b) 1. c. S. } 6 \pm 6 \text {. }\end{array}$ 
raum und die Lageveränderung der Gliedmassen, deren erhebliche Wirkung auf die Blutcirculation am Bein Braune nachgewiesen. Darum muss denn auch die Beobachtung am Lebenden, d. i. die Casuistik, zur Beurtheilung der Zweckmässigkeit der isolirten Unterbindung der Schenkelvene entscheidend bleiben.

E. v. Bergmann ${ }^{1}$ ) weist auf eine Complication hin, welche auch in Fällen von Unterbindung der Schenkelvene, in denen sonst gïnstige Bedingungen für sicheren Abfluss des Blutes, wie kräftige Herzaction oder pathologische Erweiterung der Anastomosen oder auch mangelhafter Schluss der Klappen in letzteren, gegeben sind, verhängnissvoll werden und Gangrän verursachen kann, d. i. diffuse Blutinfiltration der Gewebe. Sie kann bei geniigender Ausdehnung die Collateralen comprimiren und so den Riickfluss durch dieselben sistiren.

In gleicher Weise werden hinzutretende Wundkrankheiten, wie Phlegmone und Erysipelas, von der Unterbindungsstelle ausgehend, nicht nur durch die Gefahr entzündlicher und ödematöser Schwellung, sondern auch durch die Möglichkeit secundärer Thrombosen der Anastomosen ein günstiges Resultat der Unterbindung in Frage stellen können. Die strengste Antisepsis wird darum auch hier die Grundbedingung für einen günstigen Ausgang sein. Die in dem oben beschriebenen Falle am 2. Tage unter heftigem Fieber aufgetretenen gerötheten und überaus schmerzhaften Stellen oberhalb der Wunde sind wohl als entzïndliche Vorgänge an thrombosirten Venen der Bauchdecken zu betrachten und wahrscheinlich dadurch veranlasst, dass die Finger, welche nach Eintreten der Blutung die Wunde sogleich tamponirten, nicht ganz aseptisch waren.

Bemerkenswerth ist, dass nach der Braun'schen Statistik ron 17 isolirten Unterbindungen der V. femoralis 3 antiseptisch behandelt und diese 3 geheilt, jedoch von 15 Unterbindungen der V. und A. femoralis 8 antiseptisch behandelt waren und von diesen 8 nicht weniger denn 6 mit Gangrän und Tod endeten; ein Kranker starb an Anämie infolge von Nachblutung und nur einer wurde geheilt. In diesen 6 mit Gangrän geendeten Fällen begann kurze Zeit, meist am 1. bis 3. Tage, ja in 2 Fällen schon einige Stunden nach der Unterbindung der Arterie und Vene die Gangrän, die vom Fusse ausging und schnell fortschritt. Der unglickliche Ausgang war somit einzig die Folge der Ernährungsstörung und sprechen diese Erfahrungen, so gering: sie anch noch an Zahl sind, im Vergleich mit den 3 anti-

1) l. c. S. 230 . 
septisch behandelten und geheilten Fällen von isolirter Unterbindung der Vene sebr zu Gunsten der letzteren.

Je mehr sich das Vertrauen zu dieser durch weitere, gut verlaufene Fälle festigen wird, um so mehr wird auch - gewiss zum Nutzen des Patienten - von längeren Versuchen der seitlichen Ligatur oder Abllemmung der Vene Abstand genommen werden. Es sind im Ganzen nur 7 Fälle von seitlicher Ligatur der Vena femoralis nach ihrer Verletzung unter dem Poupart'schen Bande bekannt geworden. ${ }^{1}$ ) Die Erfolge sind keineswegs ermunternd: 4 mal erfolgte Nachblatung, ein Kranker starb an Pyämie, einer erforderte, da ein immer tiefer greifender Schorf an der Venenwand baldige Perforation befürchten liess, die doppelte Ligatur der Vene und nur ein Fall (Boyer) endete mit Heilung. - Kommt die Blutung aus einem kurz durchschnittenen Seitenast, so dass noch ein kleiner Gefässstummel dem Hauptstamm ansitzt, so ist der Versuch, die Blutung durch Unterbindung desselben zu stillen, gewiss gerechtfertigt. Ist aber der Hauptstamm selbst verletzt, so kann schon das Aufsuchen und Freilegen der verletzten Stelle äusserst schwierig und durch immer neue Blutungen gefährlich werden. Auch werden nur sehr kleine Wunden der Venenwand sich durch seitliche Ligatur schliessen lassen und ist selbst dann das Haftenbleiben derselben mehr oder weniger Glückssache. Es mögen vielleicht an der V. femoralis nicht viel mebr seitliche Ligaturen ausgefuhrt worden sein, als $\mathrm{Br}$ a u zusammengestellt hat; die Fälle aber, in denen nach Verletzung grosser Halsvenen die versuchte seitliche Ligatur nach kurzer Zeit sich wieder löste und die circuläre Ligatur nöthig machte, sind gewiss sebr zahlreich. Ist sie auch momentan geglïckt, so schwebt doch über dem Kranken noch so lange das Damoklesschwert der Nachblutung, bis die Wunde verheilt oder wenigstens mit frischen Granulationen gefüllt ist. Unter den von Braun berichteten Fällen seitlicher Ligatur trat einmal am 11. Tage und in einem zweiten Falle sogar erst am 26. Tage Nachblutung ein, die den Tod zur Folge hatte.

\section{Elftägige Anurie infolge Verstopfung beider Ureteren durch Nieren- sleine. Keine Urämie. Tod.}

L. Löwe, Kaufmann aus Hammerstein, 48 Jahr alt, litt seit 10 Jahren an Harngries, der zuweilen längere Zeit fehlte, dann wieder wochenlang auftrat. Oefters hatten sich kleine Concremente in der Harnröhre festgesetzt, die aber nach längerem Pressen durch den Harnstrahl immer spontan entfernt wurden. Dies waren die einzigen subjectiven Beschwerden;

1) Braun, Ueber den seitlichen Verschluss von Venenwunden. Archiv für klin. Chirurgie. Bd. XXVIII. Nr. 31. 
Nierenkolik war nie dagewesen. Nach Gebrauch von Marienbad im Jahre 1881 und von Karlsbad im vergangenen Jahr war in den ersten Monaten nach der Cur der Harngries spärlicher; auch die starke Corpulenz war vorübergehend gerigger geworden. Als Löwe sich, wieder auf der Reise nach Karlsbad, einige Tage in Berlin aufhielt, bemerkte er am 20. Juni 1883 völlige Suppressio urinae. Da er subjectiv gar keine Beschwerden verspürte, beunruhigte er sich niclt sonderlich darüber, blieb aber, in der Meinung, dass die Anurie durch Erkältung entstanden, zu Bett. Am 22. Juni hatte er ein unangenehmes Gefühl von Vollsein auf der Brust und suchte, nun doch durch die anhaltende Anurie beängstigt, ärztliche Hülfe. Der Arzt entleerte - trotzdem der Katheter angeblich sehr leicht in die Blase glitt nur einige Tropfen Blut; in dessen Vorschlag, in ein Krankenhaus zu gehen, willigte L. erst nach drei Tagen, "da er sich doch sonst so wohl fühle".

Status praes. bei seiner Anfnahme am 25. Juni: Der sehr fettleibige, dabei anämische Patient äussert keine Beschwerden: „Man solle ihm nur besorgen, dass er wieder Wasser lassen könne, dann wäre er sogleich gesund “. Er hat guten Appetit, wenig Durst und raucht mit Behagen seine Cigarre. Die Zunge ist nur wenig belegt, feucht. Die schlaffen Bauchdecken haben ein enormes Fettpolster; im Abdomen nirgends eine Resistenz zu fühlen, oberhalb der Symplysse keine Dämpfung, Nierengegend auf Druck unempfindlich. Am Herzen schwache, doch reine Töne, Puls 98, von niedriger Welle, A. radialis von geringer Spannung. Ein dicker silbener Katheter, bei dessen Einführen in der Regio prostatica ein knirschendes Gefühl von Rauhigkeiten bemerkbar, gelangt leicht in die Blase, entleert jedocb nur etwas Blnt; weder der Katlieter, noch eine eingeführte Steinsonde ergeben einen Fremdkörper in der Blase. Vom Mastdarm aus keine gefüllte Blase zu constatiren. Antiseptische Ausspuilung der Blase; warmes Bad. Die am nächsten Tage wiederholte Blasenuntersuchung ergibt dasselbe Resultat.

Am 27. Juni Temp. Abends 38,6 . Patient fühlt sich matt, hat keinen Appetit. Geringer Ascites.

Das Ausbleiben urämischer Erscheinungen bei achittägiger Anurie musste auf den Gedanken führen, dass das Hinderniss der Harnentleerung doch wohl in oder an der Blase gelegen, vielleicht in Form eines das Orificium urethrae vesicale verlegenden Tumors. Die steten Biutungen beim Katheterisiren, trotz ganz wegsamer Harnröhre, machten dies einigermaassen wahrscheinlich. Zudem waren sichere Anhaltspunkte, ob ein solcher Tumor und vielleicht doch eine gefullte Blase vorhanden (wogegen allerdings die Untersuchung per Rectum sprach) bei den ausserordentlich dicken Bauchdecken durch Palpation und Percussion nicht zu constatiren.

Herr Geheimrath Bardeleben führte darum am 2s. Juni die Urethrotomia externa aus. Der in die Blase eingeführte Finger stellte fest, dass ein Tumor oder Fremdlü̈rper in der ganz leeren Blase, aus der kein Tropfen Urin ansfloss, nicht vorhanden war. Es wird ein weiter Glasdrain eingelegt und in diesen ein Salicylwattebausch, zur Controle der etwa aussickernden Flüssigkeit, gebracht.

In der Nacht zum 29. Juni ist Patient sehr unruhig und lässt dünnen Stahl unter sich, der stark nach zersetztem Harn riecht. Diese unwillkïrlichen Stuhlentleerungen nelmen stetig bis znm Tode zu, haben 
in den letaten 24 Stunden eine graue Farbe und intensiv urinösen Geruch. Der Wattepfropf im Glasdrain ist stets trocken, das Wundsecret geruchlos. Der Kranke collabirt mehr und mehr, wird somnolent, ist aber immer noch leicht aus der Somnolenz zu erwecken und stirbt am 1. Juli, d. i. am 11. Tage nach Beginn der Anurie. Krämpfe waren nie aufgetreten.

Nur die Section der Bauchhöhle wird gestattet. Dieselbe (von Dr. Is rael im pathol. Institut ausgeführt) ergibt: Die Blase ist leer, fest contrahirt; ihre Schleimhant zeigt geringe hämorrhagische Flecke mit alter Pigmentirung. Der linke Ureter hat an der Grenze zwischen mittlerem und oberem Drittel eine kngelige Hervorwölbung; man fühlt an dieser Stelle einen festen Körper in ihm stecken. Dieser ist ein fast ganz runder Stein von Kirschengrösse und graugelber Farbe. An der Stelle, wo er den im Ganzen dilatirten Ureter verstopft, zeigt die Schleimhaut einen ringförmigen Substanzdefect. Der rechte Ureter ist auch erweitert, doch leer. An beiden Nieren ist die Kapsel sehr fettreich und ödematös. Beide Nieren sind stark vergrössert, von blasser Oberfläche, sehr schlaff und teigig anzufühlen. - Auf dem Durchschnitt zeigt sich die Corticalis verbreitert, weisslichgelb, die Markkegel sind hyperämisch. Beide Nierenbecken sind erweitert (das linke mehr als das rechte); ihre Schleimhant ist dunkelroth, zeigt einige Ekchymosen und ist mit trübem Schleim bedeckt. Beide Nierenbecken enthalten Nierensteine in grosser Anzahl. Dieselben, von Hirsekorn- bis Erbsengrösse (im rechten Nierenbecken befindet sich einer von Wallnussgrösse) sind rund oder etwas oval, von intensiv gelber Farbe und einzelne mit unebener drusiger Oberfläche. Sie sind ausserordentlich hart und bestehen wie die von Ilerrn Professor Salkowsky gütigst ausgeführte chemische Untersuchung ergab - ans reiner Harnsäure mit Spuren unorganischer Substanz. Die gesammelten Steine befinden sich in der Sammlung des Berliner pathologischen Institnts. - Die Serosa des unteren Theiles des Dünndarms, sowie die des Dickdarms sind stark injicirt. Die Schleimhaut zeigt keine wesentliche Veränderung bis auf das Rectum, auf dessen Falten sich diphtherische Ulcerationen finden. Die Milz ist leicht vergrössert und sehr weich.

Je länger die Anurie anhielt, um so räthselhafter wurde es, dass der Kranke sich relativ wohl fühlte, vor Allem, dass Urämie ausblieb. Erst die am 8. Tage auftretende Erscheinung, dass die reichlichen dünnen Darmentleerungen einen an Intensität mehr und mehr zunehmenden urinösen Geruch hatten, legte die Annahme nahe, dass die excrementellen Stoffe der Nieren, welche durch ihr Verbleiben im Organismus, gleichgültig in welcher Weise, Urämie zu erzeugen pflegen, durch diese profuse Darmsecretion eliminirt wurden. Dass solche complementäre Ausscheidungen durch Magen- und Darmsecret, sowie auch durch die äussere Haut stattfinden können, haben Experiment und Erfahrung bewiesen. Cl. Bernard und Barreswill, sowie Hammond ${ }^{1)}$ fanden, dass bei Hunden nach Unterbindung der Ure-

1) Heidenhain, Physiologie der Absonderungsvorgänge. 6. Abschnitt: Harnabsonderung (aus Handbuch der Physiologie von L. Hermann. Bd. V. S. 300). 
teren im reichlich gebildeten Magen- und Darmsecret reichlich Ammoniaksalze als Umsetzungsproduct des Harnstoffes auftraten, und dass die Thiere um so länger am Leben blieben, je reichlicher diese Ausscheidungen - Erbrechen und Diarrhoeen - auftraten. Am Lebenden constatirte Leube ${ }^{1}$ ), dass bei Anurie Harnstoff durch die Haut mit dem Schweiss ausgeschieden werden kann, was jedoch nicht immer Urämie verhïtet, da Bartels ${ }^{2}$ ) bei einem Urämischen das Gesicht des Kranken mit Harnstoffkrystallen bedeckt fand, nwelche besonders an den Barthaaren wie ein Reif anschossen". Andererseits bemerkt Bartels ausdrücklich - anter Hinweis auf eine von ihm beobachtete Nephritis nach Diphtherie mit mehrtägiger Anurie ${ }^{3}$ ) dass auch Fälle vorkommen, in denen trotz mehrtägiger Anurie jene complementären Ausscheidungen ausbleiben und der Tod doch ohne vorgängige Convulsionen und obne Koma nach kurzer Agone eintritt. Zugleich berichtet er ïber einen von Oeven Rees mitgetheilten Fall von völliger Anurie, welche längere Zeit bestand, ohne dass Koma oder Krämpfe sich einstellten. Der Kranke blieb im Gegentheil bis zum letzten Augenblick im vollen Gebrauch seiner Sinne. Bei der Section zeigte sich, dass die eine Niere ganz fehlte und der Ureter der einzig vorhandenen Niere durch einen Stein verstopft war. In dem noch bei Lebzeiten entnommenen Blut fand O. Rees mehr Harnstoff als in irgend einem von ihm untersuchten Falle von Bright'scher Krankheit.

Von zwei in jüngster Zeit mitgetheilten ${ }^{4}$ ) Fällen von 18- resp. 12 tägiger completer Anurie endete der erste durch Urämie. Doch trat diese erst spät ein, nachdem am 9. Tage Erbrechen erfolgt war; in den erbrochenen Massen wurde Harnstoff nachgewiesen. Bei der Section zeigten sich beide Ureteren durch Steine verschlossen. Der zweite, im Anschluss an diesen, publicirte Fall ist in seinen Erscheinungen dem oben mitgetheilten sehr äbnlich. Ein 56jähriger Mann, welcher seit einigen Jahren an Nierenkolik litt und im triiben Urin öfters Harngries fand, verspürte nach einem erneuten Kolikanfall, dass er keinen Urin lassen könne. Die Blase wurde völlig leer gefunden und auch bei wiederholtem Katheterisiren kein Tropfen Harn entleert. Dabei empfand der Kranke keine Schmerzen, nur quälte

1) Ueber den Antagonismus zwischen Harn- und Schweisssecretion. Deutsches Archiv für klin. Medicin. Bd. 7. S. 1.

2) Allgemeine Symptomatologie der Nierenkrankkeiten (aus Handbuch der speciellen Pathologie und Therapie von v. Ziemssen) S. 116.

3) 1. c. S. 125.

4) Centralblatt für Chirurgie. 1854. Nr. 3. S. 45. 
iln grosse Unruhe. Am 11. Tage trat starker Durchfall ein, wobei die entleerten graulichen Massen stark ammoniakalisch rochen. Patient wurde somnolent, collabirte und starb am 12. Tage. Die Section wurde nicht gemacht.

Im Anschluss an diese Mittheilungen wirft der Berichterstatter (Perkahl) die Frage auf, ob in äbnlichen Fällen nicht die Incision des Nierenbeckens oder Katheterisiren der Harnleiter indicirt wären. - Beide Operationen sind fraglos sehr eingreifend; denn auch das Katheterisiren der Ureteren ist nur beim Weibe ohne vorhergehenden Blasenschnitt möglich, aber auch dann nicht in ganzer Länge des Ureters ausführbar. Zudem ist die Frage schon sebwer zu entsebeiden, wann solche Operationen vorzunehmen sind. Arzt und Patient werden sich um so später dazu verstehen, je wohler sich letzterer fiihlt und um so schwerer, als Niemand vorhersagen kann, ob sich das Hinderniss des Urinabflusses nicht plötzlich spontan löst. Gehört es doch keineswegs zu den Seltenheiten, dass nach mehrtägiger absoluter Suppressio urinae infolge von Nephrolithiasis der obturirende Stein in die Blase gelangt, somit wieder Harn gelassen wird und hiermit auch die viclleicht schon eingetretenen Erscheinungen von Urämie mit einem Schlage schwinden. Die Incision des Nierenbeckens würde ausserordentliche Schwierigkeiten bieten, ja kaum ausführbar sein, wenn gar keine oder nur sehr geringe Erweiterung desselben vorhanden. Diese Erweiterung ist aber bei Leuten, welche ab und zu an Harngries leiden, sich sonst aber der besten Gesundheit erfreuen, die trotzdem aber, wie die beschriebenen Fälle gezeigt haben, durch plötzliche Verstopfung beider Ureteren in Lebensgefahr gerathen können, wenn überhaupt, so doch nur in geringer Ausdehnung vorhanden. Kommt andererseits durch behinderten Urinabfluss allmählich Hydro- resp. Pyonephrose der einen Niere zu Stande und tritt nun plötzlich durch Verstopfung des anderen Ureters völlige Anurie ein, so würde die Incision des bereits erweiterten Nierenbeckens auch nur von vorübergehendem Erfolg sein, da, wie Virchow gelegentlich der Discussion iiber einen Fall von Nierenexstirpation besonders hervorhebt ${ }^{1}$ ), fast alle Fälle von Hydronephrose sich mit interstitieller Nephritis compliciren, die meist Induration und atrophische Zustände der betreffenden Niere nach sich zieht, letztere somit mehr oder weniger functionsunfähig macht. Schon die Diagnose der Hydronephrose wird vielfach äusserst schwierig sein. Fühlt

1) $\mathrm{Vgl}$. Berliner klin. Wochenschrift. 1883. Nr. 40. Bericht über die Sitzung der Berliner medicinischen Gesellschaft rom 6. Juni 1853. 
man auch bei behindertem Urinabfluss, sei es, dass die Harnleiter von innen verstopft oder von aussen durch einen Tumor u. s. w. comprimirt werden, eine grosse, vielleicht auch fluctuirende Geschwulst in der Nierengegend und spricht dies nach dem bisherigen Krankheitsverlauf mit Wahrscheinlichkeit für eine bestehende Hydronephrose, so lässt sich diese eben nur mit einiger Wahrscheinlichkeit diagnosticiren. In einem sehr lehrreichen Fall von fünftägiger Anurie infolge Verschlusses beider Ureteren ${ }^{1}$ ) war eine starke Hervorwölbung in der rechten Lendengegend deutlich fühlbar, die nach vorn bis ungefähr zur Höhe der Spina ant. sup. reichte und absolute Dämpfung bot. Auch links war in gleicher Lage eine Geschwulst, doch von geringerem Umfang fühlbar. Die rechtsseitige Geschwulst, welche als Hydronephrose diagnosticirt worden war, wurde am 5. Tage der Anurie punctirt. Zwei Zoll oberhalb der Spina ant. sup. wurde in der Seitenwand auf der Höhe der Anschwellung in der Richtung gegen die Wirbelsäule eine Hohlnadel eingestossen und durch diese etwa $100 \mathrm{Grm}$. einer klaren, hellgelben, nicht urinösen Flüssigkeit entleert. Die Section zeigte aber, dass der Stich gar nicht das, allerdings erweiterte Nierenbecken, auch nicht die Niere getroffen hatte, sondern in die enorm vergrösserte und stark ödematöse Nierenkapsel gegangen war, die Punction also nur Flüssigkeit aus dem Oedem zu Tage gefördert hatte. Die rechte Niere war mit der Capsula adiposa fast mannskopfgross, das Fettgewebe allein $5 \mathrm{Cm}$. dick; die linke Niere nur wenig kleiner. In beiden, mässig erweiterten Nierenbecken lagen an der Abgangsstelle der Ureteren, diese völlig abschliessend, 7 resp. 12 kleine, fest aneinander gekeilte Steine und waren die Zwischenräume noch durch Harngries gefüllt, so dass sich ein fester Tampon am Anfang der Ureteren gebildet hatte. Letztere selbst waren frei. Bemerkenswerth ist, dass auch in diesem Falle Uxämie nicht eingetreten war, doch starke wässerige Darmausscheidungen infolge gereichter Drastica und reichliche Sclıweissabsonderung spontan erfolgten.

Bei Ueberlegung eines operativen Heilverfahrens verdient auch die merkwürdige Thatsache Berücksichtigung, dass einige, allerdings wenige Fälle bekannt geworden sind ${ }^{2}$, in denen complete Anurie

1) Haehner, Ein Fall von gleichzeitigem Verschluss beider Ureteren durch Nierensteine; Tod nach fünftägiger Anurie ohne urämische Erscheinungen. Berliner klin. Wochenschrift. Jabrgang 1891. Nr. 37. S. 531.

2) Ebstein, Nierenkrankheiten und Affectionen der Nierenbecken und der Ureteren (aus Handbuch der speciellen Pathologie und Therapie von v. Ziemssen) S. 158. Vgl, auch ibidem S. 172 . 
mit tödtlichem Ausgang eintrat, wo sich aber bei der Antopsie nur der eine Ureter durch Steine verschlossen, die andere Niere nebst Ureter völlig gesund fand. Um aber zu einem auch nur annähernd sicheren Schluss zu kommen, ob nur eine Niere und welche Niere "steinkrank" sei, bedarf es einer langen Beobachtung und besonders glïcklicher Umstände. Wenn während eines Nierenkolikanfalles und zwar während eines Anfalles, bei welchem ein Stein den Ureter total verstopft - statt des bisher abnormen Harns ganz klarer, heller Harn gelassen wird, so ist anzunehmen, dass die andere Niere gesund ist. ${ }^{1}$ ) Doch ist eine bereits bestehende Pyelitis der einen Seite hierbei Bedingung. - Tritt die Anurie plötzlich ein, während vorher der Harn ausser Sediment kein abnormes Ausselen oder keine weiteren abnormen Bestandtheile enthalten hat, und erfolgt die plötzliche Behinderung des Urinabflusses ohne heftigen Kolikanfall, so ist es unmöglich, zu sagen, ob und wie stark beide Nieren steinkrank sind und wo das Hinderniss, ob im Ureter oder in Nierenbecken, seinen Sitz hat.

Auch die Möglichkeit, dass vielleicht nur eine Niere vorhanden, oder es sich um eine Hufeisenniere handeln kann, wird den Entschluss eines Versuchs, das Hinderniss der Harnentleerung vom Nierenbecken aus operativ zu heben, erschweren. Drängen aber, nachdem die Diagnose: "Anurie infolge von Nierensteinen, welche die Ureteren verstopfen", möglichst sicher gestellt, entweder schwere urämische Erscheinungen oder schwerer Collaps zum Aeussersten, so wird ein Versuch, nach Eröffnung der Blase durch hohen Steinschnitt mittelst Katheter oder Bougie möglichst hoch in die Ureteren einzudringen, gewiss zu rechtfertigen sein. Sitzt ein Stein im unteren Theil des Ureters, so ist er auf diese Weise vielleicht flott zu machen. Tamponirt er aber hoch oben oder gar im Nierenbecken, so dürfte der Katheter ihn schwer erreichen. Eher würde noch ein Versuch, durch einen in den Ureter von der Blase aus möglichst hoch hinauf geschobenen Katheter oder durch eine Röhre mit weiter Oeffnung am stumpfen Ende (z. B. eine Uterusröhre) Wasser unter allmählich gesteigertem Druck in den Ureter einzutreiben, zum Ziele führen. Wie lose der einklemmende Stein sitzen kann, beweist Simpson ${ }^{2}$ ) durch das Gelingen eines höchst originellen Verfahrens, welches er in einem Falle von Harnstauung durch eingeklemmte

1) Auf Grund dieses Schlusses machte Gustav Simon seine erste Nierenexstirpation; bei der Section fand sich in der That die zweite Niere gesund.

2) Ebstein 1. c. S. 181 . 
Concremente anwandte. Er stellte nämlich den Kranken auf den Kopf, die Concremente fielen in das erweiterte Nierenbecken zuriick und der Abfluss des Harns war wieder hergestellt.

\section{Ein Fall von Luxatio humeri erecta.}

W. Glaser, Kutscher, 32 Jahr alt, wurde am 12. April 1883, als er ein sich bäumendes Pferd an der Halfter niederziehen wollte, mit erhobenen Armen von diesem emporgerissen. Das Pferd schlug, sich niederlassend, mit einem Vorderbein auf den emporgestreckten linken Arm des Glaser und riss ihn zu Boden. Er verspürte sofort heftigen Schmerz in der Schulter und vermochte den nach oben gestreckten linken Arm nicht wieder herabzubringen. Sofort zur Charité gebracht, bot er folgenden

Status: Der linke Oberarm des kräftigen und musculösen Mannes steht fast vertical in die Höhe und ist zugleich etwas nach vorn und wenig nach aussen gerichtet. Der Vorderarm ist gegen den Oberarm rechtwinklig gebeugt und rnht die Hand, die Vola etwas nach vorn gerichtet, leicht anf dem Kopf. Bei jeder Erschütterung des Körpers, z. B. beim Gehen, hält Patient vorsorglich mit der rechten Hand die linke fest. Das linke Akromion springt stark hervor und lässt sich leicht umgreifen; die Cavitas glenoidalis ist bequem abzutasten. Der Humeruskopf liegt, etwas nach hinten von der Linea axillaris, in der Höhe der Mitte des äusseren Scapularandes. Einen eigenthümlichen Anblick bietet der kräftig entwickelte M. deltoides, welcher zwei durch eine tiefe Zwischenrinne getrennte Wülste bildet. Der Abstand vom Condylus extern. Jumeri nach dem Akromion ist links um $7 \mathrm{Cm}$. kürzer als der gleiche Abstand am rechten Arm, nachdem dieser in die gleiche Stellung emporgehoben, wie der linke Arm. Aufgefordert, den linken Vorderarm za beugen, bewegt Patient nur wenig die Finger und behält unverändert den Arm im Ellenbogengelenk rechtwinklig gebeugt und die Hand anf dem leicht nach links gewandten Kopf. Sensibilitätsstörungen sind nur insofern zu constatiren, als Patient klagt, dass ihm die Finger wie taub seien. Die Reposition, auf seinen dringenden Wunsch in Narkose unternommen, gelingt ausserordentlich leicht. Sehon beim ersten Zug nach oben und aussen, unter Fixation der Schulter, springt der Kopf in die Kapsel zurück. Desault'seher Verband. In den nächsten Tagen färben sich Schulter und Brustseite grünblau, sonst traten keinerlei Störungen auf. Patient wurde auf seinen Wunseh am 9. Tage entlassen; der Aufforderung, sich nach einigen Wochen noch einmal vorzustellen, kam er leider nicht nach, so dass nicht festzustellen war, ob der Arm wieder in vollem Maasse functionsfähig geworden.

Dass eine Luxatio humeri erecta obne jede Muskelzerreissung eintreten sollte, ist nicht denkbar; zum Mindesten wird wohl die Sebne des M. supraspinatus abgerissen sein. In einem der beiden von Scharm, einem Schiiler Middeldorpf's, welcher bekanntlich diese seltene Form von Schulterluxation zuerst beschrieben ${ }^{1}$, ver-

1) Clinique européenne 1859. Bd. 2. 
öffentlichten Fälle ${ }^{1}$ ) (der Verletzte war in einem Maschinenwerk am rechten Arm durch ein Rad erst in die Höhe gerissen, dann von einer Kurbel mit grosser Gewalt getroffen worden und hatte neben der Luxatio humeri erecta eine tiefe Risswunde durch Haut und M. deltoides erlitten, die durch Pyämie zum Tode führte), ergab die Section Abreissung des Tuberculum majus und seiner drei Muskelinsertionen und Infraction des Akromion, die wohl durch directe Gewalt entstanden. Die von Seharm weiterhin angestellten 5 Versuche an Leichen ergaben jedesmal Abreissung des M. supra- und infraspinatus, zweimal auch theilweise Zerreissung der Insertion der Mm. subscapularis und pectoralis major. Die Gefässe und Nerven waren unverletzt. Zu den Versuchen wurden nur sehr magere Leichen genommen; die Muskeln Lebender sind wohl widerstandsfähiger and ibre Verletzungen daher nicht so umfangreich.

\section{Ausgedehnte Gangrän der Halsmusculatur und Lähmung des rechten Beins infolge von Neuritis ischiadica nach Kohlenoxydgasvergiftung.}

W. Kaupe, Schlächter, 28 Jahr alt, am 24. Hai 1883 aufgenommen, berichtete, dass er am 16. Nai mit einer Frauensperson, mit welcher er schon längere Zeit zusammen lebte, sich zu Bett gelegt, nachdem diese kurz vorher zur Bereitung des Abendbrodes Fener im Ofen angemacht. Als er erwachte, fühlte el stechenden Kopfschmerz und sah mit Schrecken, dass die Frau todt mit aufgedunsenem Gesicht, welches mit theilweise geplatzten und "Materie absondernden "Blasen bedeckt war, neben ihm lag. Er wollte sich erheben, stürzte aber neben dem Bett wieder bewusstlos zusammen. Er erwachte von Klopfen an der Thür, vermochte aber nicht wegen $n$ ganz gelähmten und gefihllosen Beins " aufzustehen und wurde von dem inzwischen eingedrungenen Schutzmann neben dem Bett liegend gefunden. Dies war am 18. Mai Nachmittags. Die Frau wurde am 19. Mai gerichtlich obducirt und hatte Herr Privatdocent Dr. Lesser, welcher amtlich bei der Section zugegen war, die grosse Güte, mir Folgendes über den Befund mitzutheilen: „Die Leiche war " "grünfaul " " and emphysematös. Die Körpermuskeln boten nichtsdestoweniger noch eine hellrothe Farbe dar, wie sie für CO.Vergiftung pathognomonisch ist. Das Blut war in dicken Tropfen dunkel und wurde durch Zusatz von Schwefelammonium für das unbewaffnete Auge in der nämlichen Weise verändert wie gewöhnliches Blut, zeigte aber nach $1 / 2$ stündiger Einwirkung genïgender Mengen dieses ređucirenden Mittels 2 Streifen im Grün des spektroskopischen Bildes. Eine bräunliche Flüssigkeit aus den Fäulnissblasen der Haut, welche einen enormen Gestank verbreitete, zeigte 1 Streifen in Roth, 2 in Grün, welche letztere beiden auf Zusatz von Sehwefelammonium sich ebenfalls nicht veränderten (der Streifen im Roth rührte von Methaemoglobin her, Fäulnissproduct). Somit konnte auch der positive Beweis der CO-Vergiftung auf das Unzweifelhafteste geliefert werden."

1) Scharm, De nova humeri luxationis specie. Dissert. inaug. Breslau $1 \$_{5}$ \%. 
Der p. Kaupe kam in Untersuchungshaft, da der Umstand, dass die Frau bereits in starker Verwesung, er jedoch lebend anfgefunden worden, ihn der absichtlichen Tödtung verdächtigte. Er verblieb in Haft bis zu seiner Ueberführung in die Charité; während dieser Zeit war „die Lähmung des rechten Fusses", wie er sie bereits beim Erwachen aus der Betäubung bemerkt hatte, unverändert und merkte er, unter zunehmender Schwäche, eine schmerzhafte Anschwellung des rechten Ellenbogengelenks. Er war nicht im Stande, zu gehen oder den rechten Fuss anzusetzen; auch war das Gefühl im Fuss erloschen.

In die Charité am 24. Mai 1883 überführt, zeigt der mittelgrosse, kräftig gebaute Mann völlig freies Sensorium; alle Fragen beantwortet er durchaus correct und verständig. Gesicht fieberhaft geröthet. Klagen über Schmerzen im rechten Arm, sowie Steifigkeit des Nackens und Lähmung des rechten Beines. An der rechten Nackenseite zeigt sich etwa in der Mitte zwischen Hinterhaupt und Schultergräte eine gut handtellergrosse, braunschwarz gefärbte mumificirte Hautpartie, die völlig trocken und von einem intensiv rothen Demarcationsstreifen umgeben ist. Beim Einschneiden kommt kein Tropfen Flüssigkeit heraus und dringt der Schnitt wie durch festes Leder. Erst beim Vordringen bis in die Musculatur, bei einer Tiefe von etwa $2 \mathrm{~cm}$, qnilt wenig Blut, mit schwarzbrauner Flüssigkeit gemischt, hervor. Die brandigen Stitcke werden abgetragen, darauf feuchter Sublimatverband $(0,05: 100,0)$ applicirt. - Das rechte Ellenbogengelenk ist geschwollen und schmerzhaft. Ueber dem Olekranon ist die Haut im Umfang eines Zweimarkstückes in einen schwarzen, trockenen Schorf verwandelt, in dessen Umgebung die Haut ödematös und stark geröthet ist. Unter dem Schorf ist Fluctuation und Hautemphysem zu füblen. Bei Schnitt durch den Schorf tritt unter Zischen Luft hervor. Das Olekranon ist noch von Periost bedeckt, das. Gelenk nicht geöffnet. Nach gründlicher antiseptischer Ausspülung der freiliegenden Theile wird permanente Irrigation des Armes mit Lösung von essigsaurer Thonerde angeordnet.

Zwei weitere Hautschorfe von etwa Thalergrösse finden sich noch über der 10. rechten Rippe, fast ganz in der Axillarlinie, und über dem linken Hacken. Die umgebende Haut zeigt auch einen Demarcationsstreifen, doch in geringerem Maasse als am Arm.

Das rechte Bein liegt schlaff, nach aussen gerollt, kann frei im Hüftgelenk, doch nur schwach im Kniegelenk bewegt werden. Der Fuss hängt beim Emporheben des Beins schlaff und völlig unbeweglich herab und sind Bewegungen von Fuss und Zehen ganz unmöglich. Der ganze Fuss ist anästhetisch (auch starke thermische Hautreize und elektrischer Pinsel werden nicht empfunden) bis auf einen kleinen Bezirk am inneren Fussrand, wo Nadelstiche schwach gefühlt werden. Von den Knöcheln bis etwa zur Mitte des Unterschenkels ist die Sensibilität herabgesetzt; tiefe Nadelstiche werden schwer unterschieden und ungenau localisirt. Weiter nach oben ist das Gefühl erhalten, doch angeblich etwas schwächer als links. Elektrische Reizung der Unterschenkelmuskeln sowie vom N. peroneus aus löst nicht die geringste Zuckung aus. Die Oberschenkelmuskeln reagiren auf faradischen Strom, doch erheblich schwächer als links.

In beiden Lungen zahlreiche Rhonchi.

Am Herzen schwache, doch reine Töne. Puls 112, von mittlerer Welle, 
A. radialis von geringer Spanung. Temp. 38,7. Im Harn kein Eiweiss, kein Zucker.

In den nächsten 5 Tagen schwankte die Temperatur des Morgens zwisehen 37,5 und 38,2, des Abends zwischen 38,4 und 39,0; der Puls war nie unter 110, Abends bis 130. Am Nacken schritt die Gangrän nach Peripherie und Tiefe weiter vor; fast täglich mussten nekrotische Haut- und Muskeltheile abgetragen werden. Die Eiterung war dabei gering und von schmutzig brauner Farbe. Am Arm nahm zwar die Schwellng ab, doch wurde die Tricepssehne und der hintere Theil der Gelenkkapsel gangränös, das Gelenk somit eröffnet. Ebenso drang der brandige Decubitus an der linken Ferse bis zum Fersenbein vor, während die kleine Decubitalstelle über den Rippen heilte. Die Lähmung am rechten Unterschenkel blieb unverändert, die Anästhesie erstreckte sich allmählich über den ganzen Unterschenkel; auch nahm die Kraft der Oberschenkelbenger am rechten Bein stetig ab. Beginnender Decubitus über dem rechten Trochanter major; quälender Hustenreiz; Hypostase in beiden Unterlappen. Patient wurde in ein permanentes Tymolbad gebracht.

Bei zunehmendem Verfall der Kräfte und subjectiver Euphorie zeigten die täglich sich mehrmals wiederholenden Schüttelfröste das Eintreten der Pyämie an. Die Gangrän am Nacken war zuletzt nach oben bis an das Hinterhaupt und nach unten bis zum Schulterblatt vorgedrungen; schliesslich war der Schädel zwischen Protuberantia occipit. externa und Proc. mastoid. dexter theils von Weichtheilen und Pericranium ganz entblösst, theils von brandigen Fetzen bedeckt; ferner ragten die cariösen Spitzen sämmtlicher Dornfortsätze der Halswirbel und selbst die Querfortsätze des 3.-6. Halswirbels frei hervor; die Fossa supraspinata dextr. lag frei, ohne Weichtheile und Periost, an Stelle der Nackenmusculutur der rechten Seite sah man nur eine tiefe Höhle, in deren Grund brandige Muskelfetzen sich abstiessen. 2 'Tage vor dem Tode schwoll das bis dahin intacte rechte Schultergelenk stark an und fülite sich mit jauchigem Eiter und Luft. Bei dieser enormen Zerstörung an der rechten Halsseite blieb die linke Seite rollständig frei; die Gangrän schnitt scharf an der Wirbelsäule ab. In dem gelähmten rechten Unterschenkel mehrten sich die schon anfangs ab und zu aufgetretenen reissenden Schmerzen. Am 10. Juni, d. w. am 26. Tage nach der CO-Vergiftung, trat der Tod ein.

Section am 11. Juni (Dr. Grawitz): Die Leiche zeigt in der rechten Nackengegend einen vom Hinterhaupt anfangenden, bis über die Spina Scapula hinabreichenden Defect der Hant und der darunter liegenden Musculatur, welcher nach der Mittellinie zu von den Proc. spinosi der Wirbelsäule begrenzt wird. Im Grunde des Defects ragt der obere Theil der Scapulaschuppe, vollständig vom Periost entblösst, hervor. Die sämmtlichen Processus spinosi und die Bögen der Proc. transversi des 3.-6. Halswirbels theils von Periost entblösst, theils mit schmutzig-missfarbigen Fetzen bedeckt. Der Grund der grossen Höhle ist von missfarbiger Oberfläche mit zum Theil $2 \mathrm{Mm}$. tiefem diphtherischen Infiltrat. Das rechte Schulterge lenk zeigt dickflüssigen jauchigen Inhalt, geringfügige flache Knorpeldefecte; am Collum humeri etwa in der Ausdehnung einer kleinen Bohne oberflächliche cariöse Zerstörung mit Blosslegung der Spongiosa. Das 
rechte Ellenbogengelenk ist eröffnet; die Hant an der hinteren Seite desselben in der Ausdehnung eines kleinen Handtellers zerstört. Die Knorpel des Ellenbogengelenks sind ohne Veränderung. Ferner findet sich ein annähernd zweimarkstückgrosser ovaler Hautdefect in der rechten Axillarlinie über dem unteren Rippenrand und ein etwas kleinerer, decubitaler Defect über dem rechten Trochanter. Ein gleicher Defect an der linken Ferse dringt bis auf den Calcaneus.

Beim Abpräpariren der Kopfschwarte zeigt sich dieselbe nach rechts und abwärts von der Protuberantia occipitalis externa des Hinterhauptsbeins völlig macerirt, die Ligamente zwischen Hinterhaupt und Wirbelsäıle in Brandfetzen verwandelt. Die Muskeln sind bis zum hinteren Rande des M. sternocleidom. vollständig zerstört, die Wundränder phlegmonös infiltrirt.

Auf der linken Seite des Nackens sind die Muskeln intact; doch finden sich an der Austrittsstelle der Nervenwurzeln kleine Eiterinfiltrationen.

Dicht unter der Wunde der rechten Seite liegt die Carotis mit dem N. vagus frei; der Nerv selbst ist ohne bemerkbare Veränderung.

Das Schädeldach ist von mittlerer Dicke und Schwere, der Knochen äusserst anämisch. Die ganze Tabula interna leicht verdickt. Dura straff gespannt, von sehr geringem Blutgehalt; Innenfäche glatt, glänzend weiss. Oberfläche der Pia transparent, anämisch; Basis zart, Gehirnsubstanz ausserordentlich anümisch, ziemlich feucht. Im hinteren Theil des rechten Linsenkernes steckt, von ziemlich dicker Kapsel umgeben, ein Cysticercus. Sonst keine Herderkrankung.

Pons und Medulla unverändert; auch der Wirbelkanal im Bereich der tiefen Gangrän zeigt unveränderte Rückenmarkshäute. Die Körper des 5., 6. und 7. Halswirbels sind cariös, Wirbelscheiben weich und infiltrirt.

Der rechte $N$. ischiadicus ist im Verlauf hinter dem Trochanter minor in einer Länge von gut $3 \mathrm{Cm}$. geschwollen, seine Scheide stark verdickt, hämorrhagisch infiltrirt, ober- und unterhalb intact. Auf dem Durchschnitt zeigen die einzelnen Nervenbündel ein gelbliches Aussehen und zeigen sich unter dem Mikroskop die Nervenfasern angefüllt mit fettig körnigem Detritus.

Das Herz sehr kräftig, anämisch; Klappen zart. Das lose Gewebe zwischen 0 esophagus und Wirbelsäule cadaverös verfärbt, sonst unverändert.

H als orga ne sehr blass, sonst unverändert.

Linke Lung e zeigt überall zarte, bläulich rothe Pleura, die nirgends adhärent oder getrübt ist, rechts besteht eine circumscripte, feste Verwachsung des Unterlappens; daselbst stösst man auf einen unregelmässigen, eingekapselten Gangränherd von Kirschgrösse. Weiter nach oben steckt ein metastatischer Infarct mit graurothem, nekrotischem Kern und beginnender, brandiger Demarcation. Im Uebrigen Lungengewebe lufthaltig.

Milz stark vergrössert ( $3 \mathrm{Cm}$. lang, 9 breit, 6 dick). Auf der Oberfläche zahlreiche circumscripte, blassrothe Stellen in dem lebhaft rothen $\mathrm{Pa}$ renchym erkennbar. Auf dem Durchschnitt zeigt sich der untere Umfang in beginnender, jauchiger Schmelzung, ohne dass ein bestimmter Infarct erkennbar wäre.

Leber gross und schwer. Deutliche grosse Acini mit peripherer Fettinfiltration. 
Nieren gross, etwas faulig, in der rechten steckt ein keilförmiger Infarct von dunkel schwarz-grïner Peripherie und gelbem Kern.

Im Magen galliger Inhalt; Pylorus weit und schlaff. Duodenum und Jejunum mit reichlichem, gelblichem Schleim gefült, im ganzen Darm sehr wenig Chymus.

Die mannigfachen Gangränstellen an Haut und Muskeln in diesem Fall, so verschieden sie auch an Ausbreitung und Tiefe waren, zeigten doch die auffallende Uebereinstimmung, dass sie alle an der hinteren Körperseite und, mit Ausnahme des Decubitus an der linken Ferse, sämmtlich auf der rechten Seite ihren Sitz hatten. Es liegt nahe, anzunehmen und spricht der Sitz der einzelnen Gangränstellen durchaus dafür, dass der Kranke während der langen Zeit der Bewusstlosigkeit hauptsächlich mit diesen Stellen aufgelegen und dass so zu den schweren Ernährungs - und Circulationsstörungen, welche das $\mathrm{CO}$ verursacht hat, noch mechanische Behinderung des Kreislaufs durch Druck gekommen, welche schliesslich zu dieser ausgedehnten Mortification der Haut und der Musculatur geführt.

CO vermag allerdings auch ohne solche äussere begünstigende Einflüsse tiefgreifende Veränderungen und schliesslich Nekrose der Muskeln zu erzeugen. Klebs ') beschreibt diese Veränderungen eingehend. In rasch verlaufenden Fällen zeigen die Muskeln die bekannte hell kirschrothe Färbung; dauert die Einwirkung längere Zeit oder halten auch nur die Vergiftungserscheinungen länger vor, so nehmen sie mehr und mehr einen grauen matten Ton an. Die Muskelfaser zeigt dann statt der Querstreifung eine körnige Masse, welche gleichmässig den Inhalt des Sarkolemms erfüllt. Die Körnchen sind anfangs äusserst klein, verblassen oder verschwinden grösstentheils in Essigsäure, in den stärker veränderten Bündeln werden sie grösser und glänzender und bleiben unter Einwirkung von Essigsäure ganz unverändert. $\mathrm{Zu}$ dieser parenchymatösen Degeneration kommen interstitielle entzïndliche Processe, welche in ihrer höchsten Ausbildung, vereint mit der Degeneration der Muskelsubstanz, zum Absterben des Muskels, zur wirklichen Nekrose führen. Diese Nekrose schreitet oft weiter vor und zwar dadurch, wie Klebs annimmt, dass die in Nekrose begriffenen Theile als Entzündungsreiz auf die Nachbarorgane wirken und gewisse chemische Producte liefern, welche diese wieder zum Zerfall bringen.

Auch im oben beschriebenen Fall ging die Gangrän am Halse von einer etwa bandtellergrossen Stelle nach der Tiefe und Peri-

1) Ueber die Wirkung des Kohlenoxyds auf den thierischen Organismus. Virchow's Archiv für pathol. Anatomie und Physiologie. Bd. 32. S. 4 50. 
pherie weiter, bis schliesslich fast die ganze Musculatur der rechten Halsseite zerstört war und eine riesige Höhle zwischen Hinterhaupt und Schultergräte iibrig blieb. Doch begünstigten hier sichtlich zwei Momente das Fortschreiten der Gangrän. Nach Entfernung des ersten, bereits ganz eingetrockneten Brandschorfes nämlich erschienen die darunter liegenden Muskeln keineswegs intact; denn sie hatten eine graubraune Färbung und entleerten nach dem Einschnitt eine schmutzig. rothbraune Flüssigkeit. Sie waren somit auch von vornherein schon der Nekrose mehr oder weniger verfallen, und beförderte die bald hinzutretende Sepsis in hohem Maasse ibren schnellen Zerfall und das Fortschreiten der Gangrän. Wie leicht sich Sepsis in einem mit CO vergifteten Körper entwickeln kann, lehrte ja die Gas- und Jauchebildung am Ellenbogengelenk bei unverletzter Haut. Andererseits drang die Gangrän gerade am Ellenbogengelenk, sowie an den übrigen Stellen, welche von Anfang der Beobachtung an einen Brandschorf gezeigt (über den Rippen und àn der linken Ferse), nur in die Tiefe weiter vor, in sehr geringem Maasse auch nach der Peripherie. Augenscheinlich mortificirten auch hier nur die Theile, welche dem Druck am meisten ausgesetzt waren und damit die tiefsten Ernährungsstörungen erlitten hatten. Darnach lag es näher anzunehmen, dass das Vorschreiten der Gangrän - allerdings durch den schnellen Eintritt der Sepsis begünstigt - mehr abhängig war von dem Grad der Ernährungsstörung, welchen die einzelnen Theile durch die Einwirkung der $\mathrm{CO}$ erlitten hatten, als dass die nekrotisirenden Theile eine specifische deletäre Wirkung auf die Nachbarorgane ausgeiibt hätten.

Sehr bemerkenswerth ist die durch die Autopsie gefundene Affection des rechten $\mathrm{N}$. ischiadicus, nämlich hämorrhagische Schwellung der Nervenscheide an einer circumscripten Stelle und fettige Degeneration der Nervenfasern, wodurch die Lähmung des rechten Unterschenkels erklärt ist. Muskellähmungen, namentlich der unteren Extremitäten, sind nach $\mathrm{CO}$ nicht selten beobachtet worden, doch nur sehr selten hat die Obduction eine Erkrankung der versorgenden Nerven ergeben, so dass $\operatorname{man}^{1}$ ) gemeinhin annahm, diese Lähmungen hätten in der durch das CO bewirkten Degeneration der Muskeln ibren Grund, an welcher die benachbarten Theile und besonders die Nerven nur secundär Antheil nähmen, wenn die Degeneration sich zur Nekrose steigerte. In diesem Falle aber zeigte die Musculatur des rechten Beins, ausser geringer Atrophie, keine Veränderungen, vor Allem nirgends Nekrose. Der kleine Decubitus unter dem rech-

1) Vg!. Klebs 1. c. S. 514. 
ten Trochanter entwickelte sich erst zu einer Zeit, als die Lähmung des Unterschenkels längst eine complete war, stand somit sicher in keiner Beziehung zu dieser. Der Kranke konnte schon, als er aus der Bewusstlosigkeit erwachte, nicht mehr aufstehen, da "sein rechtes Bein gelähmt war" ${ }^{\circ}$. Die Degeneration des Nerven ist also hier das Primäre und die Lähmung des Unterschenkels erst die Folge gewesen.

Einen ähnlichen Fall von Neuritis ischiadica nach CO beschreibt Leudet ${ }^{1}$ ): Einige Tage nach der Vergiftung zeigte sich erst Lähmung der Extensoren des Beines, später wurden beide untere Extremitäten, schliesslich auch die oberen und das Gesicht gelähmt. Die Section ergab Röthung und Anschwellung des rechten N. ischiadicus (an welcher Stelle?). Die übrigen Nerven und das Gehirn erschienen gesund.

CO bringt durch die chemische Veränderung des Blutes und die dadurch bedingte schwere Alteration der Ernährung die bekannten, als erstes Symptom auftretenden Störungen des Centralnervensystems hervor, es vermag aber auch direct Structurveränderungen desselben zu erzeugen. Hirnhyperämie ist bei fast allen Sectionen der durch CO Vergifteten, sowie bei allen Thierexperimenten gefunden worden. Seltener sind Blutaustritt auf und zwischen die Häute des Gehirns, sowie Hirnapoplexie, endlich Erweichungsherde des Gehirns beobachtet worden. Letztere schlossen sich entweder unmittelbar an die Intoxication an oder entwickelten sich erst längere Zeit nach der Einwirkung des $\mathrm{CO}$.

Th. Simon ${ }^{2}$ ) macht nach Zusammenstellung einer Reihe von solchen Fällen darauf aufmerksam, dass der Seh- und Streifenhtigel die Prädilectionsstellen dieser Erweichungsherde sind. Diesen Fällen ist ein weiterer von $\mathrm{Gnauck}^{3}$ ) angereiht worden, in dem nach $\mathrm{CO}$ Vergiftung Verrücktheit eintrat und wo die Autopsie beiderseits im Corpus striatum an vollkommen symmetrischen Stellen einen etwa bohnengrossen, gelben Erweichungsherd ergab. Gnauck fügt daran noch 3 weitere, ihm von Dr. Lesser mitgetheilte Sectionsbefunde von $\mathrm{CO}$ - Vergifteten, bei denen theils die innere Kapsel, theils der Linsenkern - und zwar auch beiderseits symmetrisch - Erweichungs-

1) Recherches sur les troubles des nerfs périphériques et surtout des nerfs vasomoteurs consécutifs à l'asphyxie par la vapeur de charbon. (Arch. gén. de méd. Mai 1965. p. ว13.)

2) Ueber Encephalomalacie nach Kohlengasvergiftung. Archiv für Psychiatrie. Bd. I. S. 263.

3) Casuistische Mittheilungen. Charitéannalen, Jahrgang 1891. S. 399. 
herde zeigten. Er hebt hervor, dass es nicht wunderbar sei, wenn gerade an diesen Stellen die Erweichungsherde gefunden werden, da uberhaupt in der Gegend der Seh- und Streifenhügel infolge der Vertheilung und Anordnung der Gef ässe die meisten Hämorrhagieen resp. Erweichungen entstehen.

In neuester Zeit ist über eine Lähmung des rechten Nervus trigeminus nach $\mathrm{CO}$ berichtet worden 1). Eine junge kräftige Köchin hatte nach schnell voribergehender CO-Einwirkung ein nur kurzes asphyktisches Stadium, doch trat nach wenigen Tagen eine starke schmerzhafte Schwellung des linken Beins auf, welche ihr das Gehen ganz unmöglich machte. Auch das linke Kniegelenk schwoll an. Als die Schwellung bereits ganz nachgelassen, stellte sich - 3 Wochen nach der Vergiftung - Anästhesie der rechten Stirnhälfte und der behaarten Kopfhaut bis zum Scheitel ein; ebenso waren die rechte Conjunctiva und Cornea, sowie die rechte Nasenhälfte und -Höhle nebst der rechten Zungenhälfte völlig unempfindlich. An Wange und Kinn derselben Seite war die Anästhesie "nur relativ" und bestanden keinerlei Secretions- oder vasomotorische Störungen. Ueber den weiteren Verlauf gibt Lancerea $u x$ Nichts an.

1) Lecons de Clinique médicale par Dr. Lancereaux. Paris 1883. 